\title{
Demonstration of Interference Patterns by the Random Walk of Particles
}

\author{
Igor Grabec ${ }^{1, *}$ - Nikolaj Sok ${ }^{2}$ \\ ${ }^{1}$ University of Ljubljana, Faculty of Mechanical Engeneering, Slovenia \\ 2 University of Ljubljana, Faculty of Computer and Information Science, Slovenia
}

Visualization of interference phenomena by Chladni patterns is treated. The formation of the pattern in the Young's double-slit experiment is described by a new model of driven random walk exhibited by particles bouncing on a vibrating surface. In the model the mean length of horizontal displacement is described deterministically by the wave amplitude. The presented example indicates that such formation of interference patterns can take place without any pilot-waves associated to particles. In spite od this, the pheomenon remeinds to formation of interference patterns observed at scattering of particles in quantum mechanics.

Keywords: random walk of particles, Chladni patterns, interference phenomena, Young's experiment

Highlights

- Formation of Chladni patterns caused by interfering waves on a surface is considered. For this purpose a new model of pattern formation caused by jumping of particles on a vibrating surface is introduced.

- In the model the amplitude of vibrating surface is treated as a deterministic variable, while jumping of particles is described by the Gaussian random number genarator.

- $\quad$ Numerically simulated example of Chladni pattern formation in the Young's double-slit experiment shows that the distribution of particles reveales deterministic properties of interfering waves, athough the movement of individual patterns is random.

- $\quad$ This property reminds to the situation at the double slit experiment in quantum mechanics where the trajectories of individual particles indicate random character, while their distribution reveals deterministic properties of interference.

\section{INTRODUCTION}

One of the great puzzles of nature is the wave-particle duality of quantum mechanics that was explained by De Broglie [1] and Böhm [2] by assigning to particles the so called pilot-waves. This duality had been considered an exclusive property of the microscopic world until the experiments with droplets bouncing on surfaces of vibrating liquids indicated that it might be characteristic of macroscopic particles too [3] and [4]. In these experiments droplets are accompanied by pilot-waves on the liquid surface where they can generate interference. In the quantum mechanical treatment the interference is governed by the Schrödinger equation [2], while the interference of bouncing droplets is described by the classical wave mechanics [3] and [4]. Since the presence of pilotwaves is essential in both cases, it is rather surprising that properties of interference can be observed even when particles are not accompanied by them. This article points to the corresponding example by the numerical simulation of the Chladni pattern formation caused by interfering waves in the classical Young's double-slit experiment [5].

\section{MODEL AND SIMULATION}

Chladni patterns are formed by the sand particles bouncing on vibrating surfaces and their application for the visualization of vibration modes has significantly contributed to the development of acoustics [6]. Although it is evident that during the formation of a pattern the particles move from regions of high vibration amplitude towards the calm regions at nodal lines, the analytical description of movement by bouncing remained incomplete due to its complexity [7] and [8]. To describe such movement quantitatively the trajectories of bouncing particles were statistically analyzed in recent experiments presented in the previous articles [9] to [11]. The analysis indicates that the bouncing of an individual particle corresponds to a random walk. The distribution of the horizontal displacement $\Delta \mathbf{R}(x, y)$ in this walk is Gaussian and direction independent. The standard deviation of the distribution is proportional to the amplitude of surface vibration $A(x, y)$ and characterizes the horizontal displacement length in a single bounce. This property enables a simple simulation of the pattern formation by the standard Gaussian random number generator $G$ if the distribution of vibration amplitude $A(x, y)$ is given. Comparison of simulated and actually observed patterns reveals surprisingly good agreement of 
their properties [9] to [11]. Although the bouncing is random, the governing parameter of its distribution is determined by the vibration amplitude, and therefore, the Chladni pattern formed on a vibrating surface provides information about the characteristics of the corresponding standing wave. Since a standing wave is the result of the interference between waves moving in the opposite directions, we presume that such a pattern could be applied to characterize properties of interference also in other cases. To check this presumption we simulate a random walk of particles in the Young's experiment with interfering waves from two sources. For this purpose the model of vibration driven random walk developed in the previous article is utilized [9] to [11].

In the simulation a particular step of the random walk is generated by the modified Gaussian random number generator $\left(A-A_{\mathrm{c}}\right) \times G$ with the standard deviation of the corresponding probability distribution proportional to the wave amplitude above the critical value $A_{\mathrm{c}}$ for bouncing. At the formation of Chladni patterns the critical value is usually much smaller than the wave amplitude $A_{\mathrm{c}}<<A$, and for the sake of simplicity, we discard it in the present modeling. The particle displacement in the $x$-direction during single bounce is then described by the expression: $\Delta x=A(x, y) \times G$, and similarly for the $y$-direction.

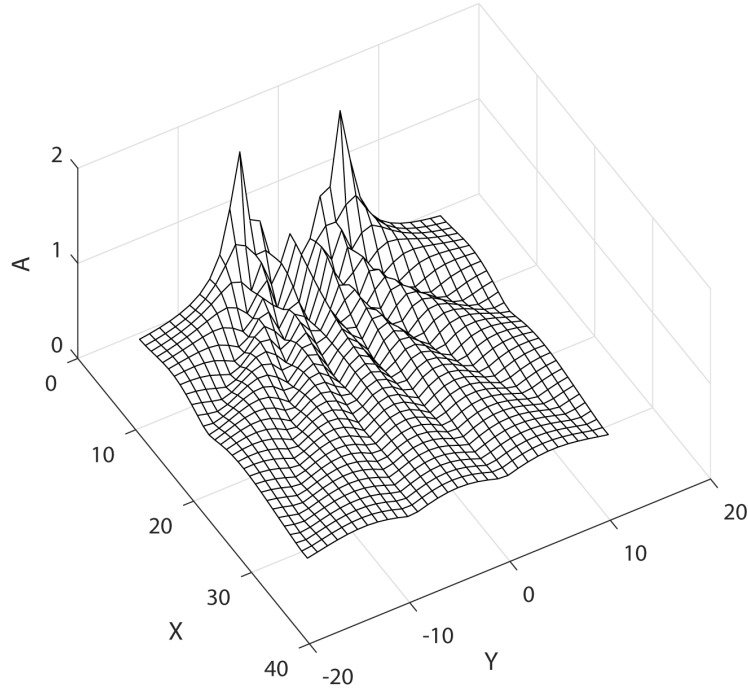

Fig. 1. The distribution of the wave amplitude $A(x, y)$ in the simulated Young's experiment with interfering waves from two sources

The distribution of the wave amplitude, that in our case equals the standard deviation of random number generator, is shown in Fig. 1, while the initial distribution of 5000 particles and the subsequent evolution of Chladni patterns in 100, 400, and 1600 bounces is displayed in Figs. 2 and 3. The changing of particle distribution is most expressive at the start of bouncing and declines with concentration of particles at low amplitude regions.

\section{DISCUSION AND CONCLUSIONS}

Figs. 2 and 3 reveal that particles move due to vibration driven random walk to the calm regions of the interference pattern. The corresponding Chladni pattern thus provides a proper basis for indicating and characterizing the interference phenomenon

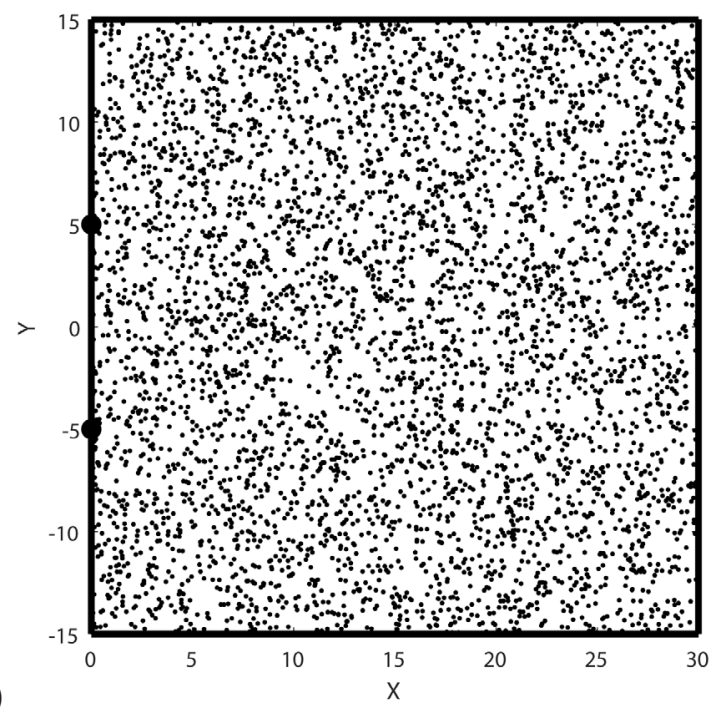

a)

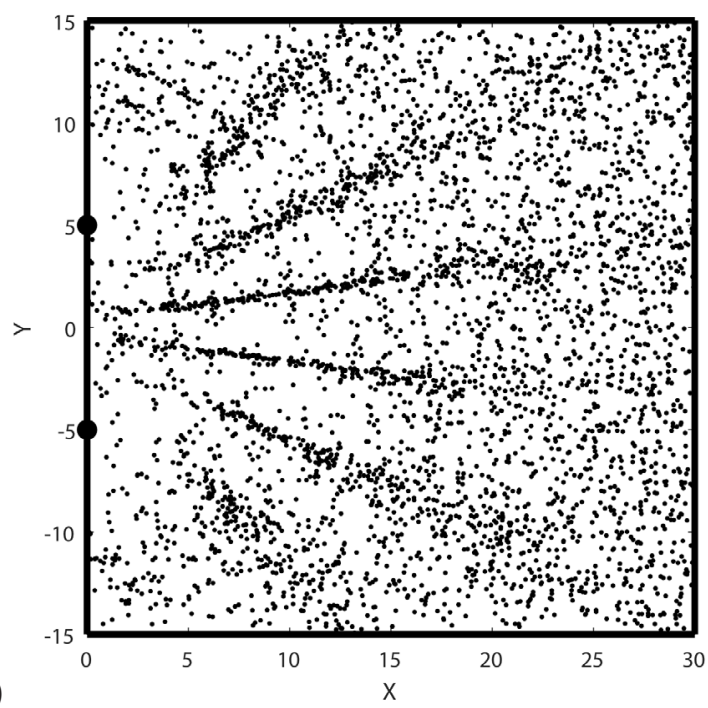

Fig. 2. a) Initial distribution of 5000 particles, and b) Chladni pattern caused by 100 bounces 
although the particles bounce randomly during its formation. However, the interference character is in this case determined only by the properties of waves on the surface and not by any pilot-waves associated with particles. The influence of this character on a particular bounce remains unexplained, but it is simply described on average by the most simple relation between the wave amplitude and the standard deviation of the horizontal displacement.

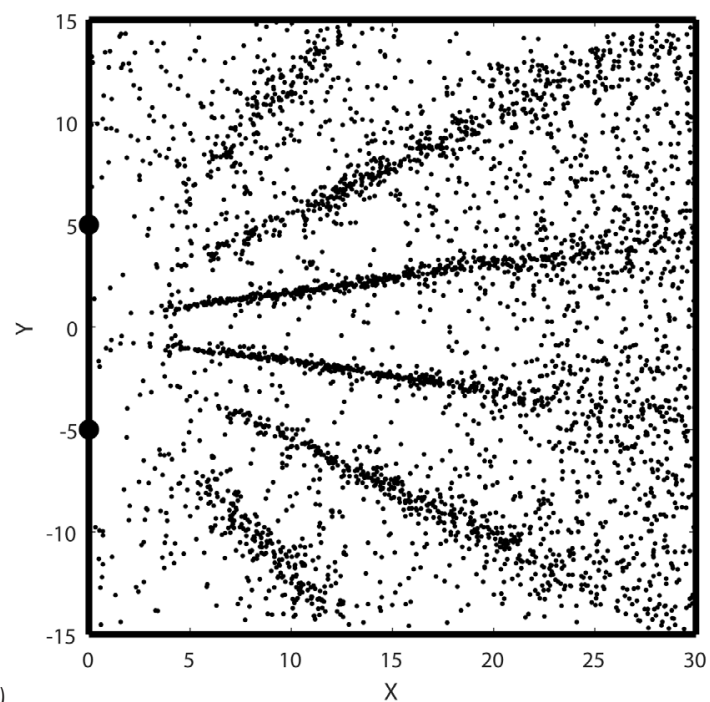

a)

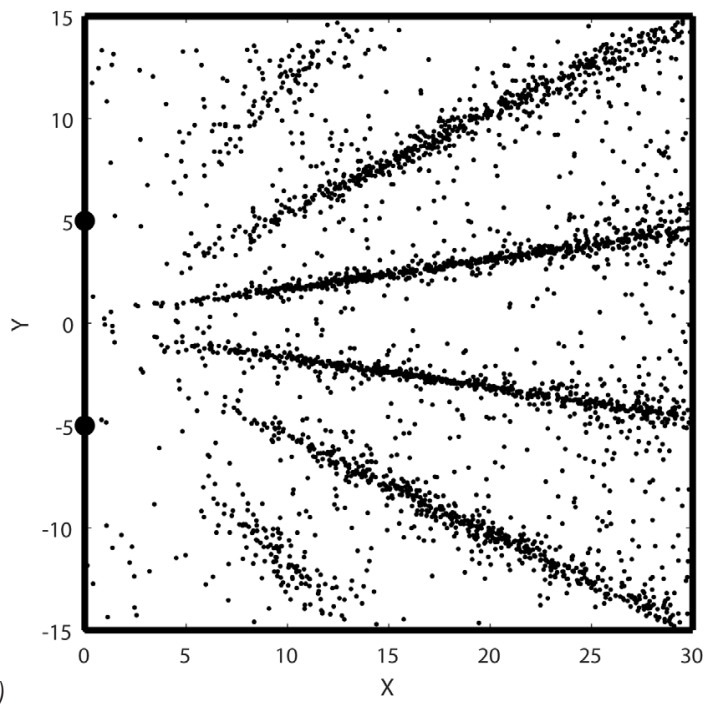

Fig. 3. a) Chladni patterns caused by 400 bounces, and b) 1600 bounces
In relation to the presented case the question arises whether it would be possible to find an example of interference phenomenon also in quantum mechanics that could be interpreted without waveparticle duality.

\section{ACKNOWLEDGEMENTS}

The authors would like to thank Prof. Tomaž Klinc for his comments and acknowledge the cooperation with the Laboratory of Synergetics at the Faculty of Mechanical Engineering, University of Ljubljana, Slovenia supported by the Slovenian Research Agency through the research core funding No. P2-0241.

\section{REFERENCES}

[1] de Broglie, L. (1953). The Interpretation of Wave Mechanics with the Help of Waves with Singular Regions, from: http:// arxiv.org/abs/1005.4534v1, accessed on 2010-05-25.

[2] Böhm, D. (1952). A suggested interpretation of the quantum theory in terms of "hidden" variables. Physical Review, vol. 85, no. 2, p. 166-179, D0l:10.1103/physrev.85.166.

[3] Couder, Y., Protière, S., Fort, E., Boudaoud, A. (2005). Walking and orbiting droplets. Nature, vol. 437, p. 208, DOI:10.1038/437208a.

[4] Couder, Y., Fort, E. (2012). Probabilities and trajectories in classical wave-particle duality. Journal of Physics: Conference Series, vol. 361, art. ID 012001, p. 1-9, D0I:10.1088/17426596/361/1/012001.

[5] Chladni, E.F.F. (1787). Entdeckungen über die Theorie des Klanges, Leipzig, D0I:10.14711/spcol/b495277. (in German)

[6] Smilansky, U., Stöckmann, H.-J. (2007). Nodal patterns in physics and mathematics -- From Chladni's seminal work to modern applications -- A historic-scientific perspective. EU Physical Journal - Special Topics, vol. 145, no. 1, p. V-VI.

[7] Lichtenberg, A.J., Lieberman, M.A. (1983). Regular and Stochastic Motion, Springer, New York.

[8] Halev, A., Harris, D. M. (2018). Bouncing ball on a vibrating periodic surface. Chaos, vol. 28, no. 9, art. ID 096103, DOI:10.1063/1.5023397.

[9] Grabec, I. (2017). Vibration driven random walk in a Chladni experiment. Physics Letters A, vol 381, no. 2, p. 59-64, DOI:10.1016/J.physleta.2016.10.059.

[10] Grabec, I., Sok, N. (2019). Formation of Chladni Patterns by Vibration Driven Random Walk of Particles. Nonlinear Phenomena in Complex Systems, vol. 22, no. 1, p. 75-83.

[11] Grabec, I., Sok, N. (2018). Statistical description of particle movement in a Chladni experiment. International Conference on "Applied Statistics", Abstracts and Program, Statistical Society of Slovenia, Ljubljana. 